\title{
REVIEW
}

\section{Updating the evidence. A systematic review of what works in preventing childhood unintentional injuries: Part 1}

\author{
E Towner, T Dowswell, S Jarvis
}

In the last 10 years there has been considerable progress in the science and art of injury prevention. The scientific study of what works in different fields of health care and health promotion has expanded, and evidence-led policy development has dominated health planning. We have collected evidence on evaluated intervention studies related to childhood injury prevention since 1992, and published reviews in 1993 and $1996 .{ }^{12}$ This paper updates this evidence. We attempt to answer three questions:

(1) Have there been any changes in the evidence relating to the effectiveness of childhood injury prevention?

(2) What additions have been made to the literature, relating to the target groups and implementation strategies of interventions?

(3) What additions have been made to the literature, relating to the ways interventions have been evaluated?

\section{Methods}

A database of primary studies has been built up over the years in the Department of Child Health in the University of Newcastle. We identified the relevant literature by a search of computerised databases (for example, BIDS, Medline, Excerpta Medica, the DHSS database, the Social Science Research Index, Web of Science, Transport Research Laboratory databases). This was supplemented by consulting with "key informants" in the field and hand searching of relevant journals, such as the journal Injury Prevention and Accident Analysis and Prevention and the reference lists of recently published books and articles.

The criteria for inclusion of studies were:

(1) They related solely or in part to the prevention of unintentional injuries.

(2) They targeted children aged 0-14 years.
(3) They described (a) primary prevention measures designed to prevent accidents, or (b) secondary prevention measures designed to reduce the impact of accidents.

(4) They had been evaluated using some outcome measure. This could include changes in mortality or morbidity, observed or reported behaviour, change in hazard, or change in knowledge.

Violence prevention studies were excluded.

Two reviewers extracted the data from each study, using a standard data extraction form (available if required). This form included sections on the aims and objectives and content of the intervention. The aims and description of the evaluation were also summarised; study design, sample size, outcome, impact, and process measures were documented. Using these extraction sheets, three reviewers independently assessed the quality of evidence for each of the studies. Studies were graded on a five point scale: good, good/reasonable, reasonable, reasonable/weak, and weak. Thus a well designed randomised controlled trial could be rated as "good" but a randomised controlled trial with a small sample size or high attrition rate of subjects, could be rated as "good/ reasonable" or "reasonable". The British National Health Service Centre for Reviews and Dissemination review guidelines were used to assess the quality of the evidence. ${ }^{3}$ Using the data extraction forms, the tables were developed; the quality rating and level of effectiveness of studies were consensus decisions by the three reviewers and a fourth member of the team was called on for additional advice.

We have summarised the key features of the studies in tables relating to the road environment, ${ }^{4-21}$ and in part 2 the home environment, ${ }^{22-32}$ the leisure environment, ${ }^{33-37}$ community based studies, ${ }^{38-42}$ and general/mass media interventions. ${ }^{43-45}$

Table 1 The road environment: traffic calming

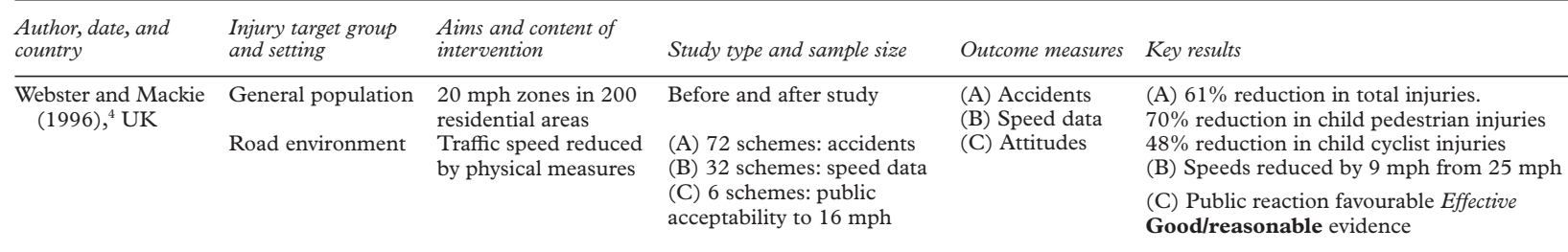


Table 2 The road environment: skills training

\begin{tabular}{|c|c|c|c|c|c|}
\hline $\begin{array}{l}\text { Author, date, } \\
\text { and country }\end{array}$ & $\begin{array}{l}\text { Injury target group } \\
\text { and setting }\end{array}$ & $\begin{array}{l}\text { Aims and content of } \\
\text { intervention }\end{array}$ & Study type and sample size & Outcome measures & Key results \\
\hline $\begin{array}{l}\text { Hazinski et al } \\
\text { (1995), } \\
\text { USA }\end{array}$ & $\begin{array}{l}5-7 \text { years } \\
\text { School based }\end{array}$ & $\begin{array}{l}\text { Children's Traffic Safety } \\
\text { Program } 10 \text { week } \\
\text { programme by teachers }\end{array}$ & $\begin{array}{l}\text { Controlled trial without } \\
\text { randomisation } \mathrm{I}=6 \text { schools } \\
(2 \text { high, } 4 \text { low income }) \mathrm{C}=1 \\
\text { school }\end{array}$ & $\begin{array}{l}\text { Observed seat belt } \\
\text { use: all occupants }\end{array}$ & $\begin{array}{l}\text { C: increased belt use: } 48.2 \% \text { to } 54.7 \% \text {. In I, } \\
2 \text { low income schools with good programme } \\
\text { implementation: } 13.4 \% \text { before } 25 \% \text { after } \\
\text { Inconclusive Reasonable/weak evidence }\end{array}$ \\
\hline $\begin{array}{l}\text { Burke et al } \\
\quad(1996),{ }^{6} \\
\text { USA }\end{array}$ & $\begin{array}{l}5-11 \text { years } \\
\text { Road environment }\end{array}$ & $\begin{array}{l}\text { Education on safe bus } \\
\text { boarding I bus stops: } \\
\text { designated safe areas } \\
\text { painted on pavements }\end{array}$ & $\begin{array}{l}\text { Randomised controlled trial } \\
\text { I: } 5 \text { bus stops C: } 4 \text { bus stops }\end{array}$ & Observed behaviour & $\begin{array}{l}\text { Children in C twice as likely to exhibit } \\
\text { unsafe behaviour ( } 75 / 174) \text { as compared } \\
\text { with children in I (38/145) Effective } \\
\text { Reasonable evidence }\end{array}$ \\
\hline $\begin{array}{l}\text { Savill et al } \\
\quad(1996),{ }^{7} \mathrm{UK}\end{array}$ & $\begin{array}{l}\text { 9/10 years assessed } \\
\text { at age } 12 . \\
\text { School/road } \\
\text { environment }\end{array}$ & $\begin{array}{l}\text { Cycling skills and road } \\
\text { safety knowledge by } \\
\text { playground and roadside } \\
\text { training }\end{array}$ & $\begin{array}{l}\text { Controlled trial without } \\
\text { randomisation. (A) Skills test } \\
I=805, C=761 \text { (B) Knowledge } \\
I=977, C=977 \text { (C) Cycling } \\
\operatorname{logs} I=442, C=366\end{array}$ & $\begin{array}{l}\text { (A) Observed } \\
\text { behaviour } \\
\text { (B) Knowledge } \\
\text { (C) Exposure to } \\
\text { cycling }\end{array}$ & $\begin{array}{l}\text { (A) } 75 \% \text { of I and } 53 \% \text { of } \mathrm{C} \text { rated "safe" } \\
\text { (B) Knowledge scores of } \mathrm{I}=8.8, \mathrm{C}=7.7 \\
\text { Reported helmet use of } \mathrm{I}=32 \%, \mathrm{C}=-20 \% \\
\text { (C) Cycling exposure similar for I and C } \\
\text { Effective Good/reasonable evidence }\end{array}$ \\
\hline $\begin{array}{l}\text { Thomson and } \\
\text { Whelan } \\
\text { (1997), }{ }^{8} \mathrm{UK}\end{array}$ & $\begin{array}{l}\text { Children } 5-7 \text { years } \\
\text { Low income } \\
\text { School based } \\
\text { Road environment }\end{array}$ & $\begin{array}{l}\text { Drumchapel Road Safety } \\
\text { Initiative Practical } \\
\text { roadside pedestrian training } \\
\text { of children by local } \\
\text { volunteers. } 3 \text { key skills } \\
\text { taught } 4-6 \text { training sessions }\end{array}$ & $\begin{array}{l}\text { Randomised controlled trial } \\
\text { Approx } 50 \text { children per year } \\
\text { in I and C for } 3 \text { specific } \\
\text { behaviours }\end{array}$ & $\begin{array}{l}\text { Observed behaviour } \\
\text { of children }\end{array}$ & $\begin{array}{l}\text { I: children's choice of "very unsafe" places } \\
\text { to cross fell from } 47 \% \text { (pre-tests) to } 12 \% \\
\text { C: children from } 50 \% \text { to } 30 \% \text { Effective } \\
\text { Good/reasonable evidence }\end{array}$ \\
\hline $\begin{array}{l}\text { Macarthur et al } \\
\text { (1998), }{ }^{9} \\
\text { Canada }\end{array}$ & $\begin{array}{l}9 / 10 \text { year old } \\
\text { children based } \\
\text { High, medium, and } \\
\text { low income schools }\end{array}$ & $\begin{array}{l}\text { "Kids CAN-BIKE" } \\
\text { Festival Playground cycle } \\
\text { skills training. Single session } \\
90 \text { minutes }\end{array}$ & $\begin{array}{l}\text { Randomised controlled trial } \\
\mathrm{I}=73 \text { children in } 3 \text { schools } \\
\mathrm{C}=68 \text { children in } 3 \text { schools }\end{array}$ & $\begin{array}{l}\text { Observed behaviour } \\
\text { Attitudes } \\
\text { Knowledge }\end{array}$ & $\begin{array}{l}\text { No significant differences between I and C. } \\
\text { Ineffective Good/reasonable evidence }\end{array}$ \\
\hline
\end{tabular}

$\mathrm{C}=$ control; $\mathrm{I}=$ intervention.

\section{Results}

We identified 42 publications for the period 1995 to 1999 which were not included in our previous systematic reviews. ${ }^{4-45}$ Of the 42 recent publications, 18 related to interventions in the road environment, 11 the home environment, five the leisure environment, five broader community based studies, and three mass media or general training studies. We rated the quality of the evidence as good/reasonable in 12 of the studies, as reasonable in 13 studies and reasonable/weak in 17 studies. The publications reviewed included eight randomised controlled trials, five of which were rated as good/reasonable evidence ${ }^{8232529}$ and three as reasonable. ${ }^{6} 1416$

Table 3 The road environment: promotion of bicycle helmets

\begin{tabular}{|c|c|c|c|c|c|}
\hline $\begin{array}{l}\text { Author, date, } \\
\text { and country }\end{array}$ & $\begin{array}{l}\text { Injury target group } \\
\text { and setting }\end{array}$ & $\begin{array}{l}\text { Aims and content of } \\
\text { intervention }\end{array}$ & Study type and sample size & Outcome measures & Key results \\
\hline $\begin{array}{l}\text { Mock et al } \\
\quad(1995),{ }^{10} \\
\text { USA }\end{array}$ & $\begin{array}{l}5-15 \text { years } \\
\text { Community-wide }\end{array}$ & $\begin{array}{l}\text { Mass media campaign } \\
\text { discount schemes. School } \\
\text { based educational activities } \\
\text { and bicycle events }\end{array}$ & $\begin{array}{l}\text { Prospective observational } \\
\text { study (A) } 8860 \text { observations } \\
\text { (B) } 466 \text { admissions }\end{array}$ & $\begin{array}{l}\text { (A) Observed } \\
\text { helmet use at } 150 \\
\text { sites } \\
\text { (B) Hospital } \\
\text { admissions }\end{array}$ & $\begin{array}{l}\text { (A) Helmet wearing when riding: } 5 \% \text { (1987) } \\
\text { to } 57 \% \text { (1993) (B) Severe head injuries (all } \\
\text { ages) } 29 \% \text { of all admissions to } 11 \% \text { Partly } \\
\text { effective Reasonable evidence }\end{array}$ \\
\hline $\begin{array}{l}\text { Parkin et al } \\
\quad(1995),{ }^{11} \\
\text { Canada }\end{array}$ & $\begin{array}{l}5-14 \text { years Low } \\
\text { income areas School } \\
\text { based }\end{array}$ & $\begin{array}{l}\text { "Be Bike Smart" Week } \\
\text { Educational and } \\
\text { promotional activities. } \\
\text { Helmets available at } \\
\text { discounted price }\end{array}$ & $\begin{array}{l}\text { Controlled trial without } \\
\text { randomisation } \mathrm{I}=3 \text { low } \\
\text { income schools } \mathrm{C} 1 \text { not clear } \\
\mathrm{C} 2 \text { (educated in previous } \\
\text { year) } 2 \text { schools }\end{array}$ & $\begin{array}{l}\text { (A) Observed } \\
\text { helmet use } \\
\text { (B) Self report } \\
\text { helmet ownership } \\
\text { use in I } \\
\text { (C) Helmets sold }\end{array}$ & $\begin{array}{l}\text { (A) Observed helmet use in I: } 4 \% \text { to } 18 \% \mathrm{C} 1 \text { : } \\
3 \% \text { to } 19 \% \text { C2: } 1 \% \text { to } 26 \% \\
\text { (B) Reported helmet ownership in I :10\% to } \\
47 \% \\
\text { (C) } 910 \text { helmets sold in I schools Inconclusive } \\
\text { Reasonable evidence }\end{array}$ \\
\hline $\begin{array}{l}\text { Farley et al } \\
\quad(1996){ }^{12} \\
\text { Canada }\end{array}$ & $\begin{array}{l}\text { 5-12 years } \\
\text { Elementary school } \\
\text { Community-wide }\end{array}$ & $\begin{array}{l}\text { Promotional activities over } \\
4 \text { years. Helmet discounts } \\
\text { and free helmets }\end{array}$ & $\begin{array}{l}\text { Controlled trial without } \\
\text { randomisation } \mathrm{I}=6087 \\
\text { observations } \mathrm{C}=2025 \\
\text { observations }\end{array}$ & $\begin{array}{l}\text { Observed helmet } \\
\text { use in variety of } \\
\text { locations }\end{array}$ & $\begin{array}{l}\text { Observed helmet use in I: } 9.6 \% \text { (1st year) to } \\
32.5 \% \text { (3rd year) In C: } 3.9 \% \text { to } 14.3 \% \\
\text { Partially effective-less effective in poor } \\
\text { municipalities Reasonable evidence }\end{array}$ \\
\hline $\begin{array}{l}\text { Ekman et al } \\
\quad(1997),{ }^{13} \\
\text { Sweden }\end{array}$ & $\begin{array}{l}0-14 \text { years } \\
\text { Community-wide }\end{array}$ & $\begin{array}{l}\text { Series of local, regional, } \\
\text { nation-wide campaigns }\end{array}$ & $\begin{array}{l}\text { Time series: } \mathrm{I}_{1}, \mathrm{I}_{2}, \mathrm{I}_{3}, 3 \\
\text { communities; } \mathrm{C}_{1}, \mathrm{C}_{2}, 2 \\
\text { communities; } \mathrm{C}_{4}=\text { Sweden } \\
\text { overall }\end{array}$ & $\begin{array}{l}\text { Hospital } \\
\text { discharge data for } \\
\text { cycling injuries }\end{array}$ & $\begin{array}{l}\text { Over } 15 \text { year period. In } \mathrm{I}_{1}: 48 \% \text { decrease in } \\
\text { bicycle injuries and } 59 \% \text { in head injuries } \\
\text { Sweden }\left(\mathrm{C}_{4}\right): 32 \% \text { decrease in bicycle injuries } \\
\text { and } 43 \% \text { in head injuries Effective } \\
\text { Reasonable evidence }\end{array}$ \\
\hline $\begin{array}{l}\text { Kim } e t a l \\
\quad(1997),{ }^{14} \\
\quad \text { USA }\end{array}$ & $\begin{array}{l}6-12 \text { years. Primary } \\
\text { health care }\end{array}$ & $\begin{array}{l}\text { Free helmet distribution } \\
\text { (I) or helmet discounts } \\
\text { (C) along with educational } \\
\text { intervention at public health } \\
\text { clinics (I and C) }\end{array}$ & $\begin{array}{l}\text { Randomised controlled trial } \\
\mathrm{I}=3 \text { clinics }(\mathrm{n}=243) \mathrm{C}=3 \\
\text { clinics }(\mathrm{n}=180)\end{array}$ & $\begin{array}{l}\text { (A) Reported use } \\
\text { of helmets }\end{array}$ & $\begin{array}{l}\text { (A) Reported helmet use: } 76 \% \text { in I and } 82 \% \\
\text { in C Partially effective for some groups } \\
\text { Reasonable evidence }\end{array}$ \\
\hline $\begin{array}{l}\text { Britt et al } \\
\quad(1998),{ }^{15} \\
\text { USA }\end{array}$ & $\begin{array}{l}3-4 \text { years. Low } \\
\text { income }\end{array}$ & $\begin{array}{l}\text { Multifaceted promotion } \\
\text { programme }\end{array}$ & $\begin{array}{l}\text { Controlled trial without } \\
\text { randomisation } \mathrm{I}=14 \text { sites, } \\
680 \text { children } \mathrm{C}=4 \text { sites, } 200 \\
\text { children }\end{array}$ & $\begin{array}{l}\text { (A) Observed } \\
\text { helmet use } \\
\text { (B) Reported } \\
\text { behaviour }\end{array}$ & $\begin{array}{l}\text { (A) I: } 43 \% \text { to } 89 \% \text { C: } 42 \% \text { to } 60 \% \text { (B) } \\
\text { Reported helmet use I: } 26 \% \text { to } 58 \% \text { C: } 36 \% \\
\text { to } 37 \% \text { Effective Reasonable evidence }\end{array}$ \\
\hline $\begin{array}{l}\text { Henrickson } \\
\text { and Becker } \\
\text { (1998), }{ }^{16} \\
\text { USA }\end{array}$ & $\begin{array}{l}10-12 \text { year old } \\
\text { children attending } \\
\text { schools. Low income } \\
\text { families }\end{array}$ & $\begin{array}{l}\mathrm{I}_{1} \text { school intervention }+ \\
\text { telephone parental } \\
\text { counselling } \mathrm{I}_{2} \text { school only } \\
\text { intervention }\end{array}$ & $\begin{array}{l}\text { Randomised controlled trial } \\
\mathrm{I}_{1}=142 \text { children } 3 \text { schools } \\
\mathrm{I}_{2}=163 \text { children } 3 \text { schools } \\
\mathrm{C}=102 \text { children } 3 \text { schools }\end{array}$ & $\begin{array}{l}\text { Self report of } \\
\text { helmet use }\end{array}$ & $\begin{array}{l}\text { Reported helmet use I } 25 \% \text { to } 39 \% \text { C: } 17 \% \\
\text { to } 20 \% \text { Partially effective Reasonable } \\
\text { evidence }\end{array}$ \\
\hline $\begin{array}{l}\text { Logan et al } \\
\quad(1998),{ }^{17} \\
\text { USA }\end{array}$ & $\begin{array}{l}5-13 \text { year old } \\
\text { children. Rural town } \\
\text { School based }\end{array}$ & $\begin{array}{l}\text { Education, helmet } \\
\text { provided, and incentive } \\
\text { scheme }\end{array}$ & $\begin{array}{l}\text { Before and after study. No } \\
\text { controls } I=2 \text { schools } 403 \\
\text { children }\end{array}$ & $\begin{array}{l}\text { Observed helmet } \\
\text { use. Self report } \\
\text { surveys }\end{array}$ & $\begin{array}{l}\text { Observed use } 3 \% \text { baseline, } 25 \% \text { one day after } \\
\text { giveaway, and } 5 \% \text { at } 9 \text { months Inconclusive } \\
\text { Reasonable/weak evidence }\end{array}$ \\
\hline
\end{tabular}

$\mathrm{C}=$ control; $\mathrm{I}=$ intervention 
Table 4 The road environment: bicycle helmet legislation

\begin{tabular}{|c|c|c|c|c|c|}
\hline $\begin{array}{l}\text { Author, date, } \\
\text { and country }\end{array}$ & $\begin{array}{l}\text { Injury target group } \\
\text { and setting }\end{array}$ & $\begin{array}{l}\text { Aims and content of } \\
\text { intervention }\end{array}$ & Study type and sample size & Outcome measures & Key results \\
\hline $\begin{array}{l}\text { Abularrage } \\
\text { (1997), } \\
\text { USA }\end{array}$ & $\begin{array}{l}\text { Children } 1-14 \text { years } \\
\text { Multiracial } \\
\text { community }\end{array}$ & $\begin{array}{l}\text { I (1) Legislation- helmet } \\
\text { use for children } 1-14 \text { years } \\
\text { (2) Campaign in month } \\
\text { before legislation. Discount } \\
\text { coupons }\end{array}$ & $\begin{array}{l}\text { Controlled trial without } \\
\text { randomisation } \mathrm{I}=14 \text { schools } \\
\mathrm{C}=12 \text { schools }\end{array}$ & $\begin{array}{l}\text { Observed helmet } \\
\text { use }\end{array}$ & $\begin{array}{l}\text { Observed helmet use: I: } 4.7 \% \text { to } 13.9 \% \text {, C: } \\
5.6 \% \text { to } 4.2 \% \text { Increased use in girls, } 5-9 \text { age } \\
\text { group Education and legislation effective } \\
\text { Legislation alone no effect on C Effective for } \\
\text { some groups Good/reasonable evidence }\end{array}$ \\
\hline $\begin{array}{l}\text { Ni et al } \\
\quad(1997),{ }^{19} \\
\text { USA }\end{array}$ & $\begin{array}{l}\text { Under } 16 \text { years } \\
\text { Community-wide }\end{array}$ & $\begin{array}{l}\text { Statewide legislation in } \\
\text { Oregon (July 1994) - all } \\
\text { children under } 16 \text { years to } \\
\text { wear cycle helmets when } \\
\text { riding on public property } \\
\$ 25 \text { fine for } \\
\text { non-compliance. Statewide } \\
\text { promotional campaigns }\end{array}$ & $\begin{array}{l}\text { 4: (i) Statewide observation } \\
\text { before: } 1610 \text {. After: } 1703 \\
\text { (ii) Observations: } 33 \text { schools } \\
\text { (iii) Survey in } 16 \text { schools } \\
\text { (iv) Telephone survey }\end{array}$ & $\begin{array}{l}\text { (A) Observed } \\
\text { helmet use in (i) } \\
\text { and (ii) (B) } \\
\text { Reported helmet } \\
\text { use and } \\
\text { knowledge in (iii) } \\
\text { and (iv) }\end{array}$ & $\begin{array}{l}\text { (A) Statewide (i) Observed use. } 25 \% \text { to } 49 \% \\
\text { Schools (ii) Observed helmet use } 20 \% \text { to } \\
56 \% \text { Schools (iii) Reported use } 15 \% \text { to } 39 \% \\
\text { Helmet ownership } 51 \% \text { to } 76 \% \text { Telephone } \\
\text { survey (iv) Reported helmet use } 37 \% \text { to } \\
66 \% \text { Effective Good/reasonable evidence }\end{array}$ \\
\hline
\end{tabular}

$\mathrm{C}=$ control; $\mathrm{I}=$ intervention.

Table 5 The road environment: seat belt legislation

\begin{tabular}{|c|c|c|c|c|c|}
\hline $\begin{array}{l}\text { Author, date, } \\
\text { and country }\end{array}$ & $\begin{array}{l}\text { Injury target group } \\
\text { and setting }\end{array}$ & $\begin{array}{l}\text { Aims and content of } \\
\text { intervention }\end{array}$ & Study type and sample size & Outcome measures & Key results \\
\hline $\begin{array}{l}\text { Margolis et al } \\
\text { (1996), }{ }^{20} \\
\text { USA }\end{array}$ & $4-15$ years & $\begin{array}{l}\text { North Carolina Child } \\
\text { Passenger Safety Law (Oct } \\
\text { 1985). Children } 4-15 \text { years } \\
\text { required to use safety belts } \\
\text { when front seat passengers }\end{array}$ & Time series data $1990-95$ & $\begin{array}{l}\text { Police reports } \\
\text { mortality and } \\
\text { morbidity data }\end{array}$ & $\begin{array}{l}42 \% \text { decline in deaths and serious injuries } \\
\text { Effective Reasonable evidence }\end{array}$ \\
\hline $\begin{array}{l}\text { Williams et al } \\
\text { (1997), }{ }^{21} \\
\text { USA }\end{array}$ & $\begin{array}{l}\text { Day care and } \\
\text { elementary schools }\end{array}$ & $\begin{array}{l}\text { "Click it or Ticket" } \\
\text { program Statewide } \\
\text { enforcement of seat belt } \\
\text { laws. Education at schools }\end{array}$ & $\begin{array}{l}\text { Controlled trial without } \\
\text { randomisation } \mathrm{I}=2 \text { schools } 2 \\
\text { day care centres } \mathrm{C}=1 \\
\text { elementary school } 1 \text { day } \\
\text { care centre }\end{array}$ & $\begin{array}{l}\text { (A) Observed } \\
\text { seatbelt use (B) } \\
\text { Child restraint } \\
\text { violations }\end{array}$ & $\begin{array}{l}\text { (A) I schools correct restraint use } 36 \% \text { to } \\
64 \% \text { and } 49 \% \text { to } 71 \% \text {. C schools } 36 \%-43 \% \text {. } \\
\text { I day care } 71 \%-76 \%, 60 \%-75 \% \text {. C day care } \\
52 \%-70 \% \text { (B) } 1025 \text { child restraint violations } \\
\text { Partially effective Reasonable }\end{array}$ \\
\hline
\end{tabular}

$\mathrm{C}=$ control; $\mathrm{I}=$ intervention.

\section{Summary of findings related to effectiveness}

INTERVENTIONS TO REDUCE INJURES IN THE

ROAD ENVIRONMENT

One new study evaluated the effect of areawide environmental change on traffic speeds and cyclist and pedestrian injuries (table 1). This study evaluated the impact of traffic speed reduction zones (20 mile per hour ( $\mathrm{mph}$ ) zones) in 200 residential areas in the UK. ${ }^{4}$ This study did not include control data but it did report on a large range of schemes and included a long period of data collection. It strengthens the evidence related to reducing accidents to vulnerable road users provided by earlier urban safety schemes. ${ }^{46}$

Five articles examined the issue of pedestrian and cyclist training as a means of increasing knowledge and promoting behavioural change (table 2). These additions to the literature mean that there is now increased, but still limited, evidence that bicycle training schemes can improve safe riding behaviour and that education on safe bus boarding can achieve some positive effect. Thomson and Whelan's study suggests that children's choice of safe places to cross can be improved by intensive roadside training. ${ }^{8}$

The promotion of bicycle helmets was addressed in a relatively large number of papers. Eight studies evaluated the effects of educational campaigns (table 3 ) and two examined the impact of legislation on helmet use by children (table 4 ). These studies suggest that educational campaigns and legislative change can achieve some positive effect on behaviour. There is more limited evidence relating to the effect of such interventions on injury rates.
There is increasing evidence that legislation requiring the restraint of children in cars has a positive effect. Two papers addressed this issue (table 5).

This project has been funded by England's NHS Executive National R\&D Programme in Mother and Child Health (CH10-21)

1 Towner E, Dowswell T, Jarvis S. Reducing childhood accidents. The effectiveness of health promotion interventions: a literature review. London: Health Education Authority, 1993.

2 Towner E, Dowswell T, Simpson G, et al. Health promotion in childhood and young adolescence for the prevention of unintentional injuries. London: Health Education Authority, 1996.

3 Arblaster L, Entwistle V, Lambert M, et al. Review of the research on the effectiveness of health service interventions to reduce variations. (CRD report 3.) York: University of York, NHS Centre for Reviews \& Dissemination, 1995.

4 Webster D, Mackie A. Review of traffic calming schemes in 20 mph zones. (TRL report 215.) Crowthorne: Transport Research Laboratory, 1996.

5 Hazinski M, Eddy V, Morris J. Children's traffic safety program: influence of early elementary school safety education on family seat belt use. F Trauma 1995;39:10638.

6 Burke G, Lapidus G, Banco L, et al. Evaluation of the effectiveness of a pavement stencil in promoting safe behavior
among elementary school children at school bus stops. Pediatrics 1996;97:520-30.

7 Savill T, Bryan-Brown K, Harland G. The effectiveness of child cycle training schemes. (TRL report 214.) Crowthorne, Berkshire: Transport Research Laboratory, 1996.

8 Thomson J, Whelan K. A community approach to road safety education using practical training methods. The safety education using practical training methods. The
Drumchapel project. (Road safety research report No 3.) Drumchapel project. (Road safety research
London: Department of Transport, 1997.

9 Macarthur C, Parkin P, Sidky M, et al. Evaluation of a bicyMacarthur C, Parkin P, Sidky M, et al. Evaluation of a bicy-
cle skills training program for young children: a randcle skills training program for young children:
omized controlled trial. Inj Prev 1998;4:116-21.

10 Mock C, Maier R, Boyle E, et al. Injury prevention strategies to promote helmet use to decrease severe head injuries at a level 1 trauma centre. F Trauma 1995;39:29-33.

11 Parkin P, Hu X, Spence L, et al. Evaluation of a subsidy program to increase bicycle helmet use by children of low-income families. Pediatrics 1995;96:283-7.

12 Farley C, Haddad S, Brown B. The effects of a 4-year program promoting bicycle helmet use among children in Quebec. Am ₹ Public Health 1996;86:46-51.

13 Eckman R, Schelp L, Welander G, et al. Can a combination of local, regional and national information substantially increase bicycle-helmet wearing and reduce injuries? Experiences from Sweden. Accid Anal Prev 1997;29:321-3.

14 Kim A, Rivara FP, Koepsell T. Does sharing the cost of a bicycle helmet help promote helmet use? Inj Prev
bict 1997;3:38-42.

15 Britt J, Silver I, Rivara F. Bicycle helmet promotion among low income preschool children. Inj Prev 1998;4:280-3. 
16 Hendrickson S, Becker H. Impact of a theory based intervention to increase bicycle helmet use in low income children. Inj Prev 1998;4:126-31.

17 Logan P, Leadbetter S, Gibson R, et al. Evaluation of a bicycle helmet giveaway program: Texas 1995. Pediatrics 1998 ; 101:578-82.

18 Abularrage J, Deluca A, Abularrage C. Effect of education and legislation on bicycle helmet use in a multi racial population. Arch Pediatr Adolesc Med 1997;151:41-4.

$19 \mathrm{Ni} \mathrm{H}$, Sacks J, Curtis L, et al. Evaluation of a statewide bicycle helmet law via multiple measures of helmet use. Arch Pediatr Adolesc Med 1997;151:59-65.

20 Margolis L, Bracken J, Stewart J. Effects of North Carolina's mandatory safety belt law on children. Inj Prev 1996;2:32-

21 Williams A, Wells J, Ferguson S. Development and evaluation of programs to increase proper child restraint evaluation of programs to increase
use. $\mathcal{F}$ Safety Res $1997 ; 28: 197-202$.

22 Bablouzian L, Freedman E, Wolski K, et al. Evaluation of a community based childhood injury prevention program. Inj Prev 1997;3:4-16.

23 Clamp M, Kendrick D. A randomised controlled trial of general practitioner safety advice for families with children under 5 yea

24 Thompson R, Edwards P, Jarvis S, et al. Childhood accidents: is it time to prescribe safety equipment? Community Practitioner 1998;71:138-40.

25 Kendrick D, Marsh P, Fielding K, et al. Preventing injuries in children: cluster randomised controlled trial in primary care. BMF 1999;318:980-3.

26 Mallonee S, Istre G, Rosenberg M, et al. Surveillance and prevention of residential-fire injuries. $N$ Engl $\mathcal{F} \mathrm{Med}$ 1996;335:27-31.

27 McConnell C, Leeming F, Dwyer W. Evaluation of a fire-safety training program for preschool children. Fournal of Community Psychology 1996;24:213-27.

28 Shults R, Sacks J, Briske L. Evaluation of three smoke detector promotion programs. Am f Prev Med 1998;15: detector

29 DiGuiseppi C, Slater S, Roberts I, et al. The "Let's Get Alarmed" initiative: a smoke alarm giveaway programme. Inj Prev 1999;5:177-82.

30 King L, Thomas M, Gatenby K, et al. "First Aid for Scalds" campaign: reaching Sydney's Chinese, Vietnamese, and Arabic speaking communities. Inj Prev 1999;5:104-8.

31 Rodgers $\mathrm{G}$. The safety effects of child-resistant packaging for oral prescription drugs: two decades of experience. FAMA 1996;275:1661-5.

32 Liller K, Craig J, Crane N, et al. Evaluation of a poison prevention lesson for kindergarten and third grade students. Inj Prev 1998;4:218-21.
33 D'Argenio P, Cafaro L, Santonastasi F, et al. Capodanno Senza Danno: the effects of an intervention program on fireworks injuries in Naples. Am $\mathcal{f}$ Public Health 1996;86: $84-6$.

34 Malinowska-Cieslik M, Borne B. Prevention of mushroom poisoning of children: effectiveness of a community-based school education programme. Health Education Research 1998;13:13-23.

35 Withaneachi D, Meehan T. Promoting safer play equipment in primary schools: evaluation of an educational campaign. Health Promotion fournal of Australia 1998;8:125-9.

36 Bennett E, Cummings P, Quan L, et al. Evaluation of a drowning prevention campaign in King County, Washington. Inj Prev 1999;5:109-13.

37 Sibert J, Mott A, Rolfe K, et al. Preventing injuries in public playgrounds through partnership between health services and local authority: community intervention study. BMF 1999:318:1595.

38 Svanström L, Ekman R, Schelp L, et al. The Lidköping accident prevention programme - a community approach to preventing childhood injuries in Sweden. Inj Prev 1995; 1:169-72.

39 Day L, Ozanne-Smith J, Cassell E, et al. Latrobe Valley better health project. Evaluation of injury prevention program 1992-1996. (Report No 114.) Melbourne: Monash University Accident Research Centre/Victorian Health Promotion Foundation, 1997

40 Petridou E, Tolma E, Dessypris N, et al. A controlled evaluation of a community injury prevention project in two Greek islands. Int $\mathcal{F}$ Epidemiol 1997;26:173-9.

41 Lindquist K, Timpka T, Schelp L, et al. The WHO safe community program for injury prevention: evaluation of the impact on injury severity. Public Health 1998;112:385-91.

42 Ytterstad B, Smith G, Coggon C. Harstad injury prevention study: prevention of burns in young children by community based intervention. Inj Prev 1998;4:176-80.

43 Gielen A, Dannenberg A, Ashburn N, et al. Teaching safety: evaluation of a children's village in Maryland. Inj Prev $1996 ; 2: 26-31$.

44 Sundelin C, Rosmussen F, Berfenstram R, et al. Information through television: does it promote child safety? Inj Prev 1996;2:36-40.

45 Marsh P, Kendrick D. Injury prevention training: is it effective? Health Education Research 1998;13:47-56.

46 Mackie A, Ward H, Walker R. Urban safety project. 3. Overall evaluation of area wide schemes. (TRRL research report No 263.) Crowthorne, Berkshire: Transport and Road Research Laboratory, 1990. 